\title{
Role of Plasma Neutrophil Gelatinase Associated Lipocalin as a New Biomarker
}

\section{TO THE EDITOR}

I read with interest the study of Raluca Fodor et al., on the significance of Plasma Neutrophil Gelatinase-Associated Lipocalin (NGAL), as an early biomarker for acute kidney injury in critically ill patients, published recently in issue no.4/2015 of JCCM journal [1]. In this well-written and interesting study, the authors successfully demonstrated that in critically ill patients, increased levels of NGAL predict, with a good sensitivity and specificity, the development of acute kidney injury within forty-eight hours of admission to an ICU. However, as no information is presented on the aetiology of the acute kidney injury, we believe that the article raises interesting and still un-elucidated hypotheses on the pathophysiological substrate of the systemic release of NGAL in patients with critical conditions.

One of the clinical scenarios accompanied by a significant increase in NGAL levels is represented by acute coronary syndromes or cardiogenic shock. It is well-known, that in patients with cardiogenic shock, acute renal failure represents one of the most significant predictor of an adverse outcome [2] and in several recently published studies, the role of NGAL has been reported as predictor of disease severity and worse outcomes in relation to acute coronary syndromes or cardiogenic shock $[3,4]$. It is also important to note that patients with acute coronary syndromes, presenting at an emergency department, usually undergo imperative angiographic examination, and acute kidney injury is a known common complication following coronary angiography associated with contrast material administration. In patients with ST-segment elevation, acute myocardial infarction treated with primary percutaneous intervention, elevated serum levels of NGAL represented an independent predictor of all-cause mortality and major adverse cardiac events [4].

NGAL is released in circulation by damaged renal tubular cells, in the first hours after the acute kidney injury, analogously to troponin release in the first hours following acute myocardial injury [4]. At the same time, recent data suggest that NGAL may also be in- volved in the development of atherosclerotic process via modulation of matrix metalloproteinase, an important mediator of plaque vulnerability in atherosclerosis, and increased amounts of NGAL have been identified in unstable atherosclerotic plaques [5].

In this context, elevation of NGAL levels, in critically ill patients with acute myocardial infarction, could be attributed to the development of acute kidney injury in cardiogenic shock accompanying severe infarctions, but also to severe atherosclerosis. In my opinion, in patients with severe infarctions and deteriorated haemodynamic status, it is not sufficiently clear whether the elevated NGAL levels reflect the severity of acute kidney disease or the severity of the acute coronary event.

Therefore, it would be very interesting, in my opinion, to perform a sub-analysis of this study, in which NGAL levels would be analyzed in the subgroup of critically ill patients with acute myocardial infarction as compared with the subgroup of critically ill patients with other etiologies of acute kidney injury.

\section{Ota Hlinomaz*}

International Clinical Research Center, St. Anne's University Hospital Brno, Brno, Czech Republic

Received: 12 December 2015 / Accepted: 16 December 2015

\section{REFERENCES}

1. Fodor R, Grigorescu B, veres M, et al. Plasma neutrophil Gelatinase Associated Lipocalin (NGAL) - Early Biomarker for Acute Kidney Injury in Critically III Patients. J Crit Care Med. 2015;4:154-61.

2. Fuernau G, Poenisch C, Eitel I, et al. Prognostic impact of established and novel renal function biomarkers in myocardial infarction with cardiogenic shock: A biomarker substudy of the IABP-SHOCK II-trial. Int J Cardiol. 2015;191:159-66.

3. Torregrosa I, Montoliu C, Urios A, et al. Urinary KIM-1, NGAL and L-FABP for the diagnosis of AKI in patients with acute coronary syndrome or heart failure undergoing coronary angiography. Heart Vessels. 2015;30:703-11.

4. Lindberg S, Pedersen SH, Mogelvang R, et al. Prognostic utility 
Available online at: www.jccm.ro

of neutrophil gelatinase-associated lipocalin in predicting mortality and cardiovascular events in patients with STsegment elevation myocardial infarction treated with primary percutaneous coronaryintervention. J Am Coll Cardiol. 2012;60:339-45.
The Journal of Critical Care Medicine 2016;2(1) • 57

5. Hemdahl AL, Gabrielsen A, Zhu C, et al. Expression of neutrophil gelatinase-associated lipocalin in atherosclerosis and myocardial infarction. Arterioscler Thromb Vasc Biol. 2006;26:136-42 